\title{
Fabrication of 3-D Hollow Metallic Probes Designed for Plasmonic Field Enhancement
}

\author{
In-Yong Park ${ }^{1,2}$, DongHyup Lee ${ }^{1}$, Joonhee Choi ${ }^{1}$, Cheolsu Han ${ }^{2}$, Sang Jung Ahn ${ }^{2}$ and Seung-Woo Kim ${ }^{1}$ \\ ${ }^{1 .}$ Ultrafast Optics for Ultraprecision Group, Korea Advanced Institute of Science and Technology \\ (KAIST), Daejeon, 305-701, South Korea \\ 2. Center for Advanced Instrumentation, Division of Industrial Metrology, Korea Research Institute of \\ Standards and Science (KRISS), Daejeon 305-340, South Korea
}

Nano-scale probes are used to overcome the diffraction limit of light in microscopic imaging, high resolution spectroscopy, and plasmonic nanofocusing $[1,2]$. For each application, it is essential to establish a suitable fabrication method to precisely produce the probe shape as optimized in the design stage. Wet etching is preferably adopted for shaping nano-scale tips, but the fabrication flexibility is limited since the taper angle is determined by the etching rate that varies with the crystal plane [3]. Here, we demonstrate a flexible approach using the focused ion beam (FIB) process, which permits milling 3D hollow plasmonic metallic probes with complicated cross-sectional profiles and arbitrary taper angles.

The metallic probe considered here was designed as a waveguide to induce surface plasmon polaritons (SPPs) so as to tightly focus the incident laser field on the sub-wavelength scale at the apex of the probe tip. The intensity enhancement is expected to reach to a factor of 100 , but the actual degree of field enhancement is greatly affected by the geometrical shape of the probe tip. It is therefore necessary to carefully define the physical shape of the probe so as to induce an efficient SPPs interaction with the incident photons. If the hollow void of the probe is linearly tapered, the energy of propagating SPPs can be accumulated gradually, thereby enhancing the intensity of a driving laser field near the probe tip. In order to optimize the important dimensions of the tapered waveguide, we adopted the finite-difference time-domain (FDTD) method to calculate the field enhancement of the designed waveguide shape. The FDTD method predicts the temporal evolution of the spatial field distribution with a single calculation run covering a wide spectral range. Silver is used for the tip material as free electrons in silver are in excellent resonance with visible or near infrared lasers. Through trial and error simulations, the four geometrical parameters of the waveguide were finally determined as follows; tapered angle $\left(\theta=14^{\circ}\right)$, cross-sectional ellipticity $(\varepsilon=0.5)$, output diameter $(\mathrm{D}=100 \mathrm{~nm})$ and waveguide length $(\mathrm{L}=9 \mu \mathrm{m})$ when the input laser is assumed to have a $800 \mathrm{~nm}$ center wavelength with $10 \mathrm{fs}$ pulse duration.

For fabrication of the tapered waveguide optimized by FDTD simulation, we chose the focused ion beam (FIB) milling process. The hollow core of the waveguide is constructed on a commercial nearfield scanning optical microscope (NSOM) probe for the sake of incorporating it with AFM (Atomic Force Microscopy) system. Fig. 1 shows the fabrication sequence in detail. As the initial step of fabrication, the hollow pyramid inside the NSOM probe is filled with platinum by FIB deposition to secure a flattened rectangular area where the tapered waveguide is to be built. After that, a silver layer thicker than the target waveguide length is deposited onto the whole probe by e-beam evaporation. The FIB process is then used to carve the desirable pattern on the target substrate by loading a bitmap image file. It is not easy to produce the 3-D tapered waveguide structure, being optimized as above, at once just with a single bitmap. Thus, we divided the hollow core of the waveguide into 31 slices of thin elliptical cylinders of which sizes are consecutively becoming smaller. The elliptical cross-section is then 
removed in the direction normal to the silver layer so that a total of 31 horizontally flat laminates is etched one by one to a depth of $300 \mathrm{~nm}$ from the inlet surface towards the exit aperture. We polished the bottom end surface of the NSOM probe until the exit aperture of the waveguide reaches an intended diameter. Finally, the probe tip is sharpened by applying the FIB process along the lateral direction from outside. The top view and the cross-sectional view of the fabricated waveguide are shown in Fig. 2 together with the reference spots used for compensation of the beam drift.

In summary, we designed and fabricated the plasmonic waveguide inside the commercial NSOM probe with the aid of FDTD simulation and FIB milling process. The method introduced in this paper can be applicable to various nanostructures by altering either the configuration of bitmap images or the FIB operating conditions. Furthermore, without any difficulties, the hollow waveguide probe could be installed easily inside the existing AFM and NSOM systems for bio-imaging and surface metrology applications requiring plasmonic field enhancement.

References:

[1] I.-Y. Park et al., Nat. Photon. 5 (2011), p. 677.

[2] F. De Angelis et al., Nat. Nano. 5 (2010), p. 67.

[3] J. S. Hyun et al., Mater. Sci. Eng. B, 149 (2008), p. 292.

[4]This work was supported by the Creative Research Initiative Program, the National Space Laboratory Program and the Basic Science Research Program (2010-0024882) funded by the National Research Foundation of the Republic of Korea.

(a)

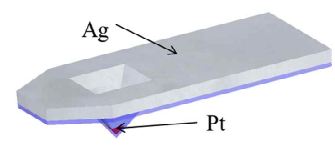

(c)

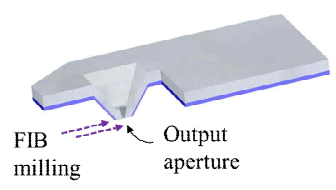

(b)

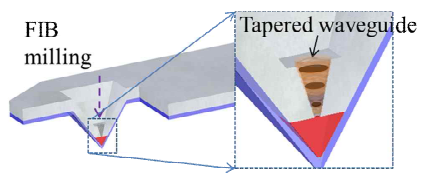

(d)

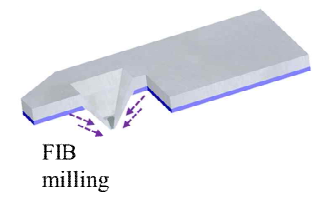

Figure 1. Fabrication procedure of the hollow elliptical metallic probe. (a) Platinum and silver deposition by e-beam evaporation; (b) The tapered waveguide carved by consecutive FIB milling processes; (c) Output aperture fabrication by lateral slicing; (d) Tip sharpening.

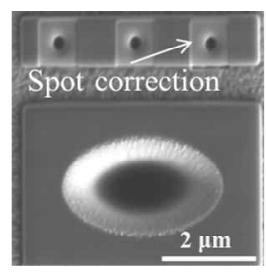

(a)

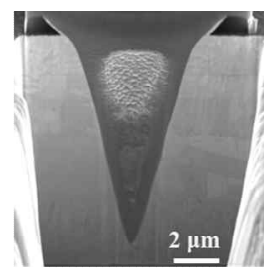

(b)

Figure 2. SEM (Scanning Electron Microscope) images. (a) Top view of the fabricated tapered waveguide and reference spots used for compensation of beam drift; (b) Cross-sectional view of the fabricated hollow waveguide. 